\title{
Bacterial Toxins Escape the Endosome by Inducing Vesicle Budding and Collapse
}

Ashweta Sahni and Dehua Pei*

Department of Chemistry and Biochemistry and Ohio State Biochemistry Program, The Ohio State University, 484 West 12th Avenue, Columbus, OH 43210, USA.

Correspondence to: pei.3@osu.edu

\section{Supporting Information}

\section{This PDF file includes:}

Fig. S1. Time-lapse confocal microscopy showing another DT-induced vesicle budding-andcollapse event in a HeLa cell.

Fig. S2. Vesicle budding-and-collapse events induced by lower concentrations of DT.

Fig. S3. Formation of tubular structures on endosomes during the endosomal escape of DT.

Fig. S4. Time-Lapse confocal microscopic images showing DT-induced vesicle budding and collapse from an enlarged endosome.

Fig. S5. Time-lapse confocal microscopic images showing three additional NleC-induced vesicle budding-and-collapse events.

Fig. S6. Time-lapse confocal microscopic images showing two vesicle budding-and-collapse events induced by lower concentrations of NleC.

Fig. S7. Formation of tubular structures at the endosomal membrane during the endosomal escape of NleC.

Captions for Movies S1 to S6

Other Supplementary Materials for this manuscript include the following:

Movies S1 to S6 

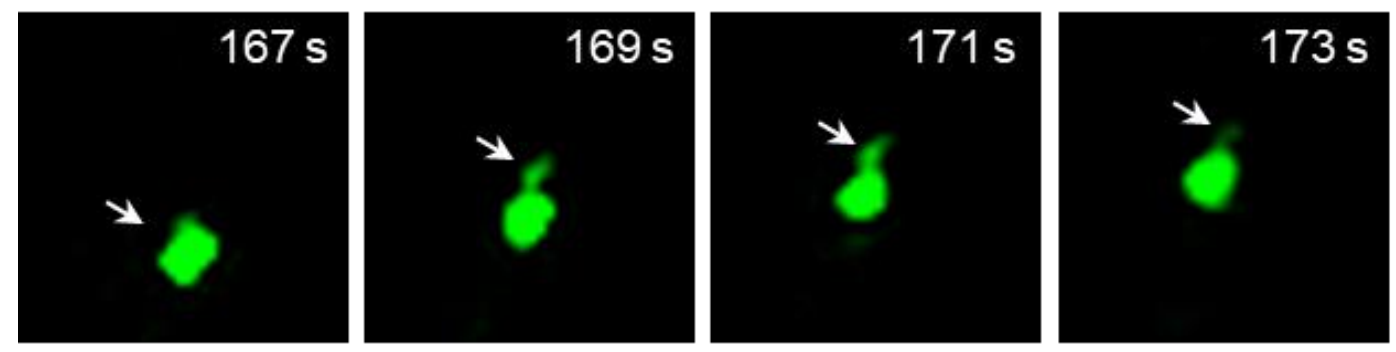

PSTopFluor
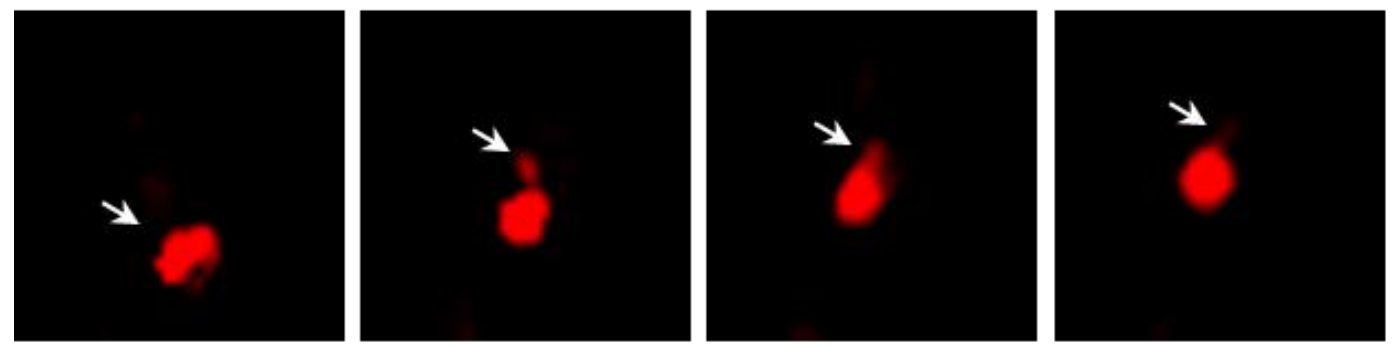

DTPHAb
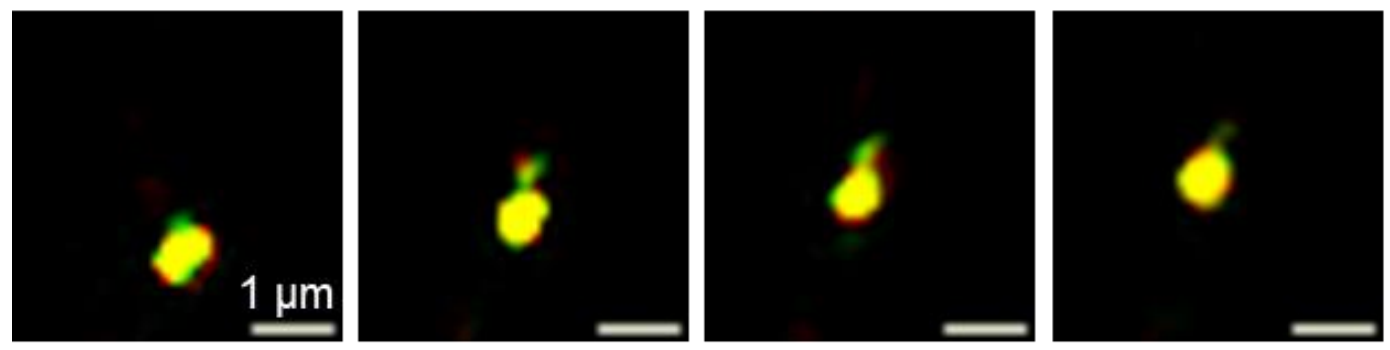

Merge

Fig. S1. Time-lapse confocal microscopy showing another DT-induced vesicle budding-andcollapse event in a HeLa cell. Cells were incubated with PS ${ }^{\text {TopFluor }}(1.5 \mu \mathrm{M}$, green channel) for 10 min, washed with DPBS, and treated with $\mathrm{DT}^{\mathrm{pHAb}}(5 \mu \mathrm{M}$, red channel) for $30 \mathrm{~min}$. Cells were washed with DMEM to remove any remaining extracellular $\mathrm{DT}^{\mathrm{pHAb}}$, supplemented with unlabeled DT $(5 \mu \mathrm{M})$, and imaged at 2-s intervals. Images of a single endosome during the period of 167$173 \mathrm{~s}$ after the initiation of imaging are displayed. The white arrows indicate the budded vesicle and its remnants after collapse. Scale bars $=1 \mu \mathrm{m}$.

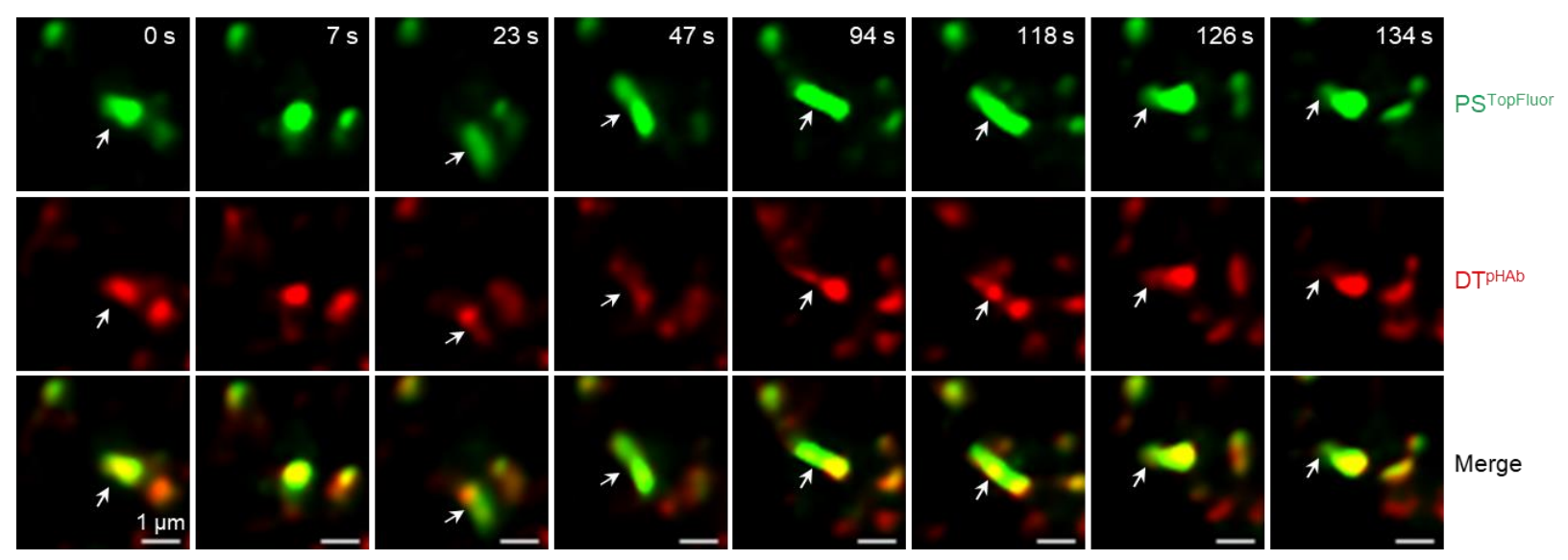

Fig. S2. An additional vesicle budding-and-collapse event induced by a lower concentration of DT. HeLa cells were incubated with $\operatorname{PS}^{\text {TopFluor }}(1.5 \mu \mathrm{M})$ and $\mathrm{DT}^{\mathrm{pHAb}}(200 \mathrm{nM})$ for $30 \mathrm{~min}$, washed 
with DMEM, and imaged by time-lapse confocal microscopy every $\sim 8 \mathrm{~s}$. The white arrows in indicate the endosome that underwent vesicle budding and collapse. Scale bars $=1 \mu \mathrm{m}$.
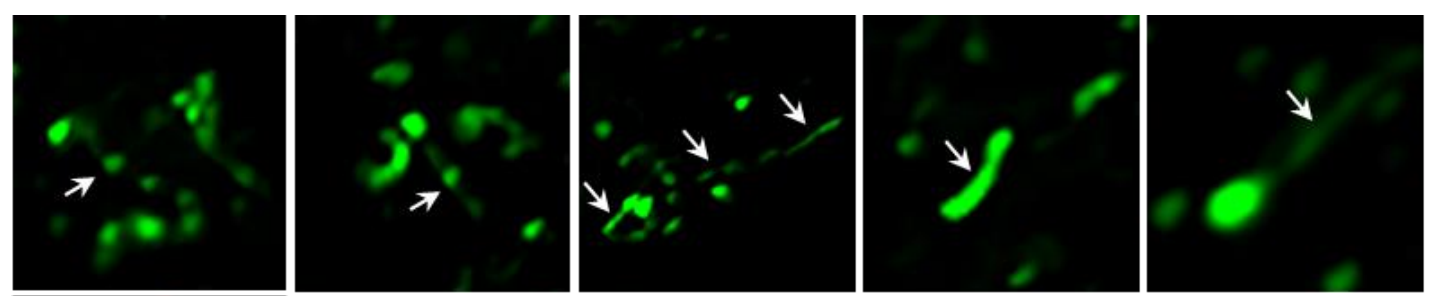

PSTopFluor
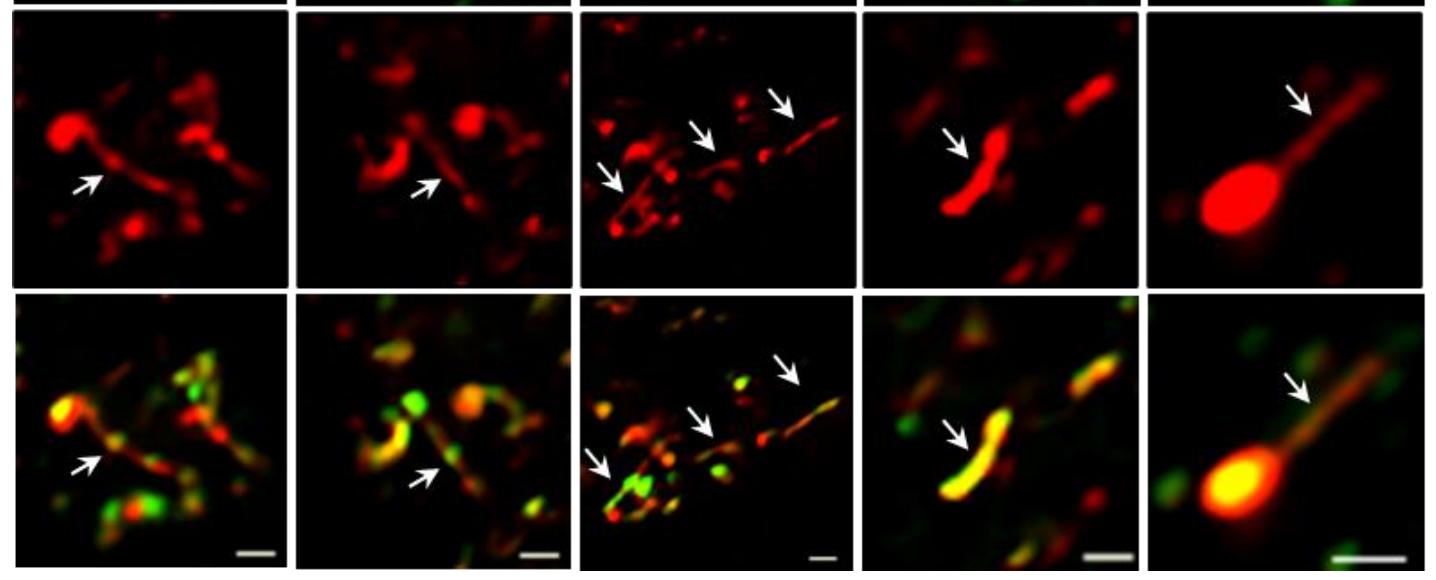

DTPHAD

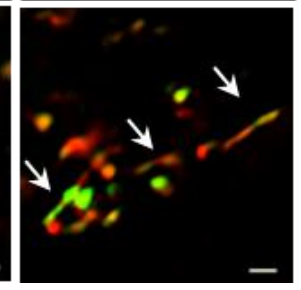

Merge

Fig. S3. Formation of tubular structures on endosomes during the endosomal escape of DT. HeLa cells were incubated with PS ${ }^{\text {TopFluor }}(1.5 \mu \mathrm{M})$ for $10 \mathrm{~min}$, washed with DPBS, and treated with $\mathrm{DT}^{\mathrm{pHAb}}(0.2$ to $1.0 \mu \mathrm{M})$ for $30 \mathrm{~min}$. The cells were washed with DMEM to remove any extracellular $\mathrm{DT}^{\mathrm{pHAb}}$ and imaged by confocal microscopy. Images shown are from different cells. Scale bars = $1 \mu \mathrm{m}$. 


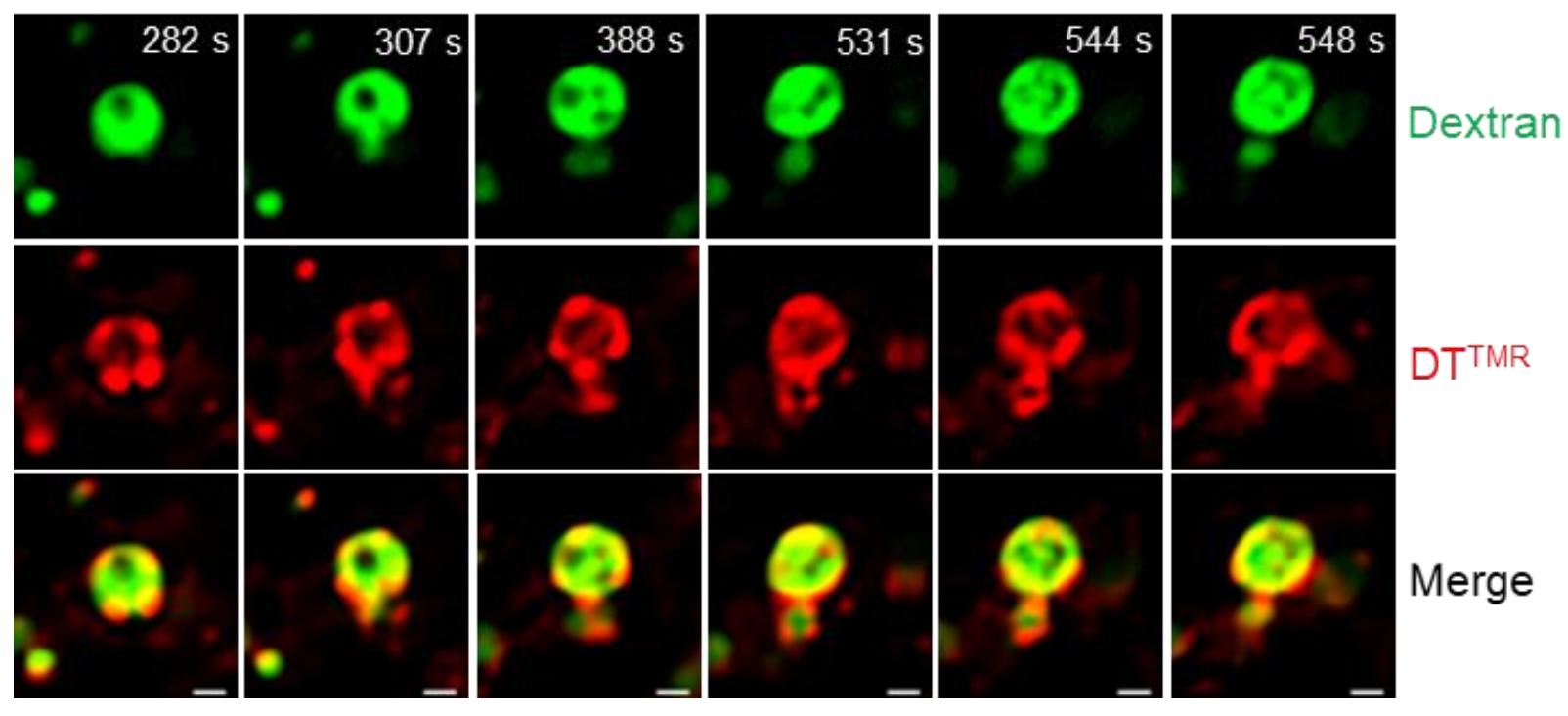

Fig. S4. Time-Lapse confocal microscopic images showing DT-induced vesicle budding and collapse from an enlarged endosome. HeLa cells were pretreated with YM201636 (800 nM) for 2 $\mathrm{h}$ and DT ${ }^{\mathrm{TMR}}(5 \mu \mathrm{M})$ and Dextran ${ }^{\text {Alexa488 }}(50 \mu \mathrm{g} / \mathrm{mL})$ were added. After incubation for another 30 min, the cells were washed and imaged by live-cell confocal microscopy at 4-s intervals. A representative vesicle budding-and-collapse event (between $282-548$ s) from a single endosome is shown. Scale bars $=1 \mu \mathrm{m}$. 


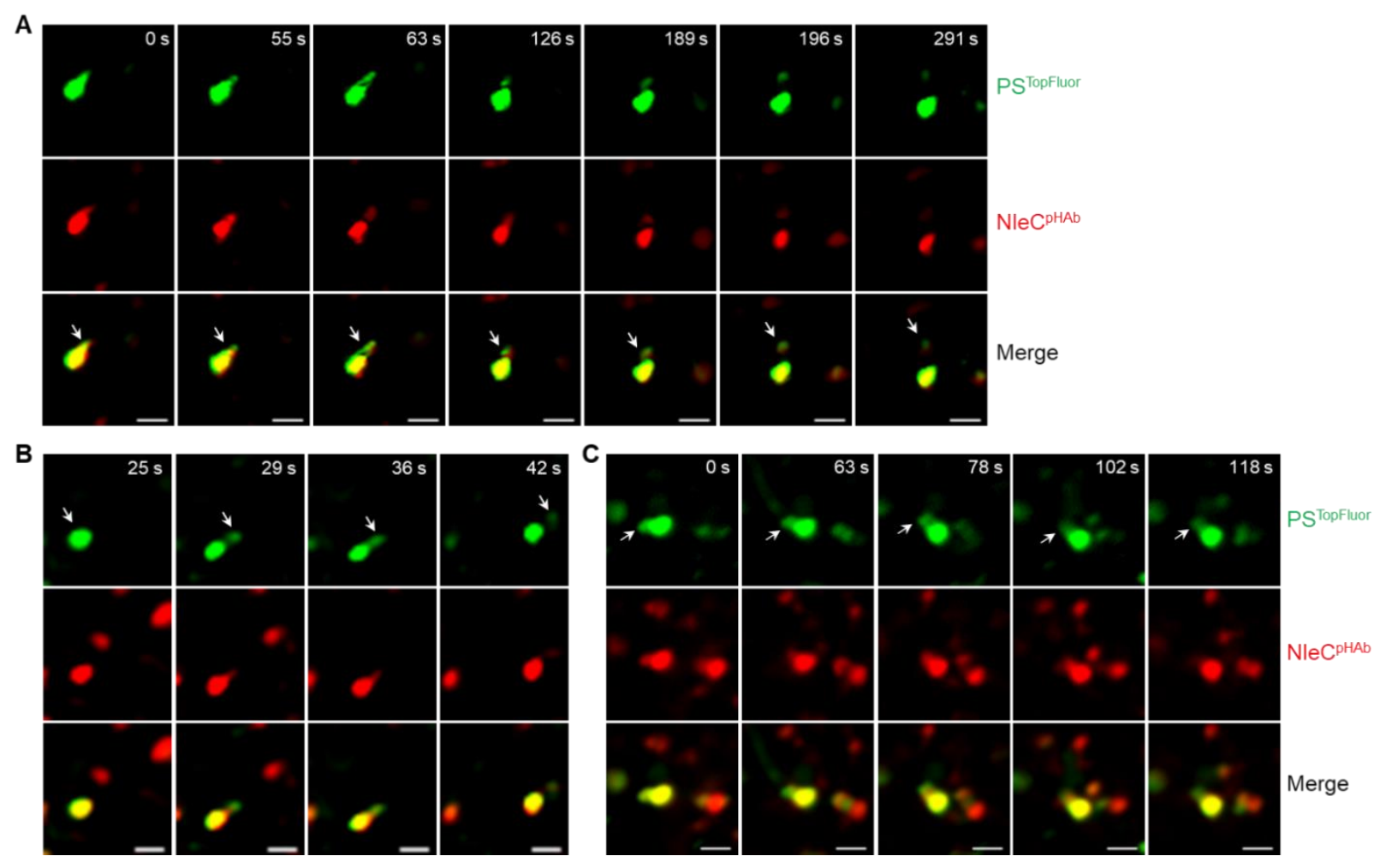

Fig. S5. Time-lapse confocal microscopic images showing three additional NleC-induced vesicle budding-and-collapse events. HeLa cells were incubated with $\operatorname{PS}^{\text {TopFluor }}(1.5 \mu \mathrm{M})$ and $\mathrm{NleC}^{\mathrm{pHAb}}(5$ $\mu \mathrm{M}$ ) for $30 \mathrm{~min}$, washed with DMEM, and imaged every 2-8 s. Scale bars $=1 \mu \mathrm{m}$. 

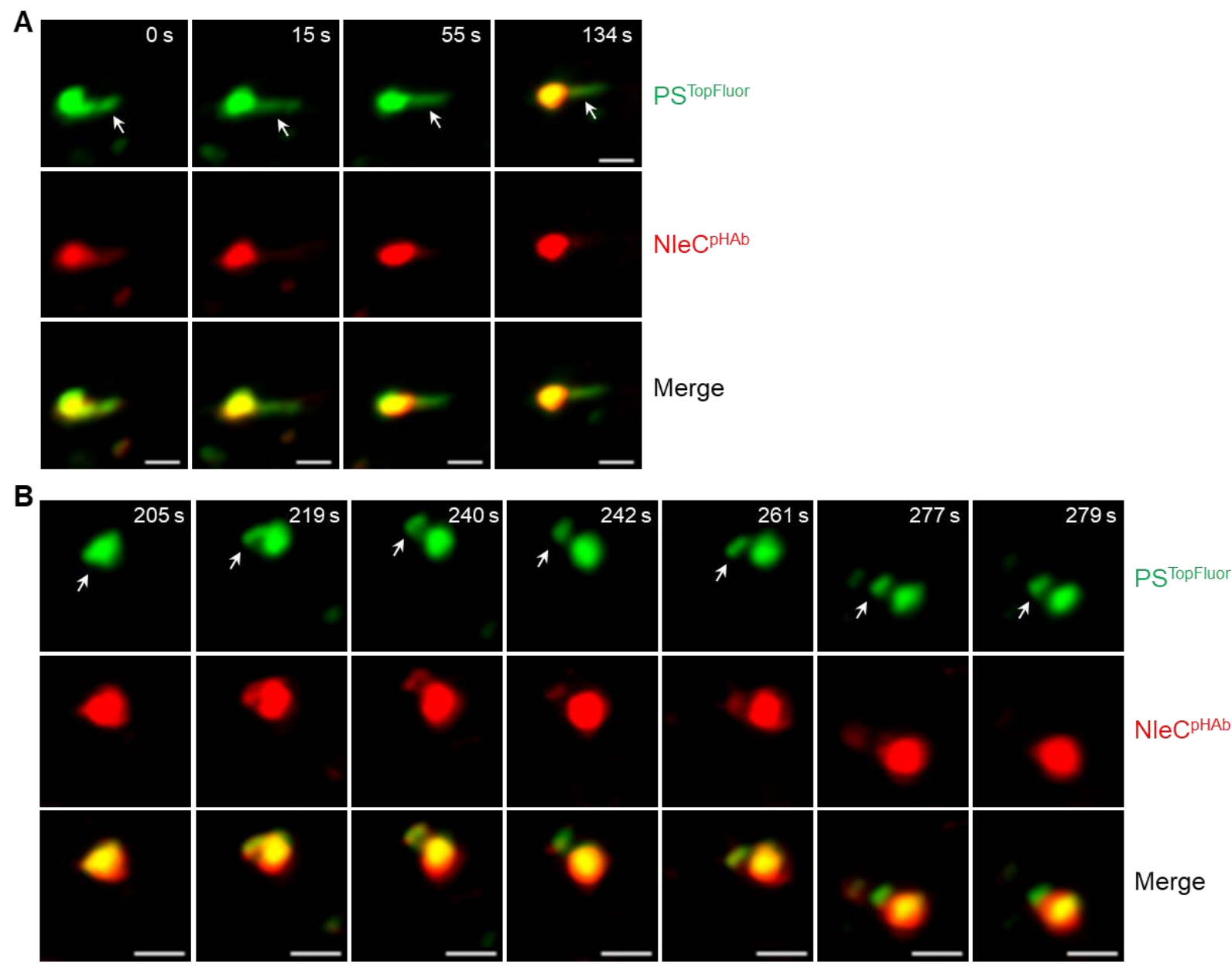

Fig. S6. Time-lapse confocal microscopic images showing two vesicle budding-and-collapse events induced by lower concentrations of NleC. HeLa cells were incubated with PS ${ }^{\text {TopFluor }}$ (1.5 $\mu \mathrm{M})$ and $\mathrm{NleC}^{\mathrm{pHAb}}(500 \mathrm{nM})$ for $60(\mathrm{~A})$ or $30 \mathrm{~min}(\mathrm{~B})$, washed with DMEM, and imaged every 28 s. Scale bars $=1 \mu \mathrm{m}$. 


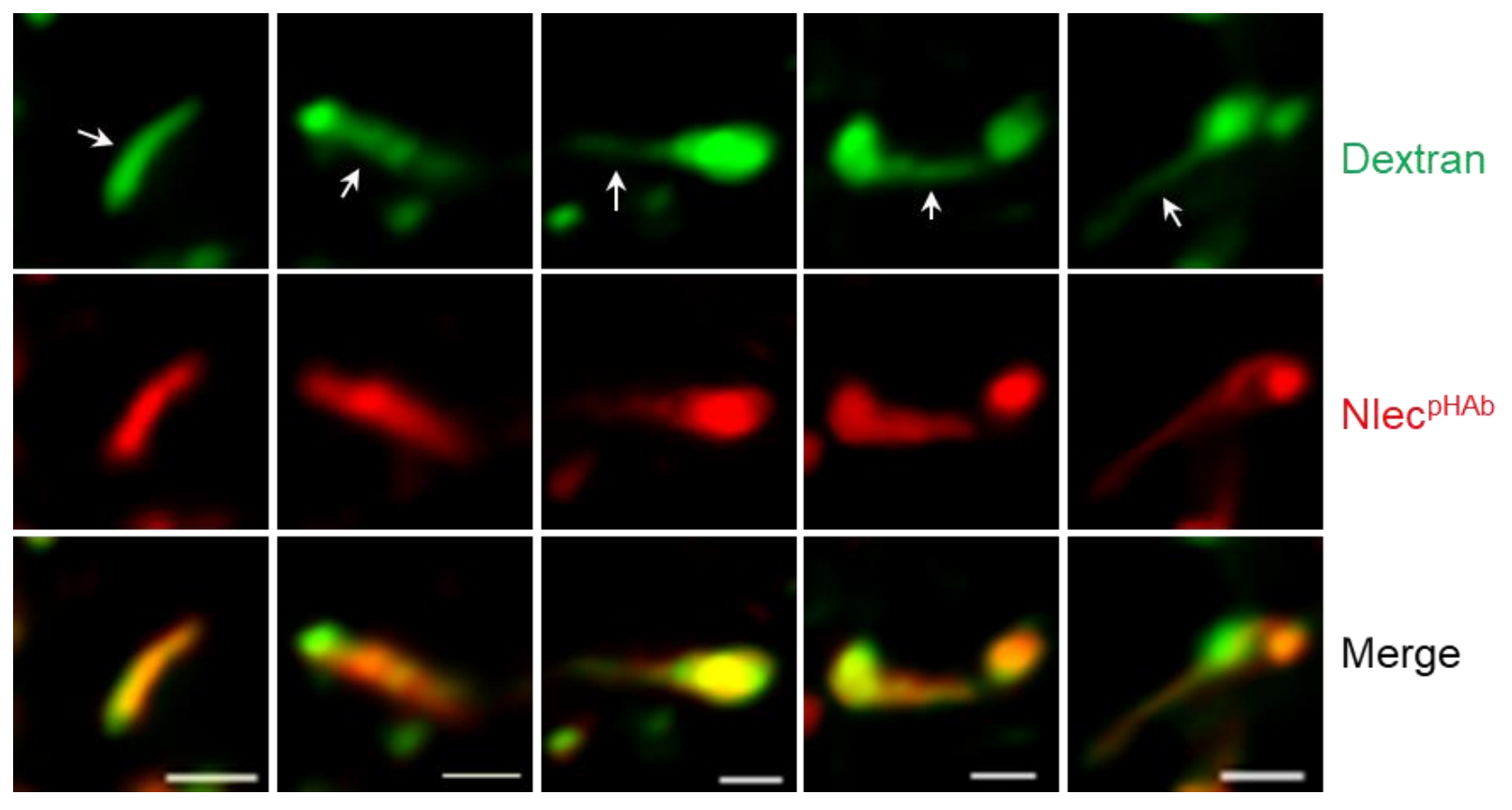

Fig. S7. Formation of tubular structures at the endosomal membrane during the endosomal escape of NleC. HeLa cells were incubated with $\operatorname{PS}^{\text {TopFluor }}(1.5 \mu \mathrm{M})$ and $\mathrm{NleC}^{\mathrm{pHAb}}(5$ or $0.5 \mu \mathrm{M})$ for 30 min, washed with DMEM, and imaged. Images of different endosomes in different cells are shown. Scale bars $=1 \mu \mathrm{m}$. 
Movie S1. A DT-induced vesicle budding-and-collapse event in a HeLa cell. Cells were incubated with $1.5 \mu \mathrm{M}$ PS ${ }^{\text {TopFluor }}$ (green channel) for $10 \mathrm{~min}$, washed with DPBS, and treated with $5 \mu \mathrm{M} \mathrm{DT}^{\mathrm{pHAb}}$ (red channel) for 30 min. Cells were washed with DMEM to remove $\mathrm{DT}^{\mathrm{pHAb}}$, treated with $5 \mu \mathrm{M}$ unlabeled DT, and imaged by confocal microscopy every $4 \mathrm{~s}$. The video consists of time-lapse images of a single endosome (boxed in Fig. 1B), 23 min after the addition of unlabeled DT and 4-48 $\mathrm{s}$ after the initiation of imaging. Panels from left to right represent the merge, $\mathrm{PS}^{\mathrm{TopFluor}}$ (green), and $\mathrm{DT}^{\mathrm{pHAb}}$ signals (red), respectively. A budded vesicle emerged from an endosome (indicated by an arrow) at $28 \mathrm{~s}$, completely separated from the endosome at $32 \mathrm{~s}$, and collapsed at $40 \mathrm{~s}$. Scale bars, $1 \mu \mathrm{m}$.

Movie S2. A DT-induced vesicle budding-and-collapse event in a HeLa cell. Cells were incubated with $1.5 \mu \mathrm{M}$ PS ${ }^{\text {TopFluor }}$ (green channel) for $10 \mathrm{~min}$, washed with DPBS, and treated with $5 \mu \mathrm{M} \mathrm{DT}^{\mathrm{pHAb}}$ (red channel) for 30 min. Cells were washed with DMEM to remove $\mathrm{DT}^{\mathrm{pHAb}}$, treated with $5 \mu \mathrm{M}$ unlabeled DT, and imaged by confocal microscopy every $2 \mathrm{~s}$. The video consists of time-lapse images of a single endosome (shown in Figure S1), during the period of 167-173 s after the initiation of imaging, undergoing endosomal escape. Panels from left to right represent the merge, PS $^{\text {TopFluor }}$ (green), and DT ${ }^{\mathrm{pHAb}}$ signals (red), respectively. Scale bars $=1 \mu \mathrm{m}$.

Movie S3. Tubule formation during the endosomal escape of DT. HeLa cells were incubated with PS ${ }^{\text {TopFluor }}(1.5 \mu \mathrm{M})$ for 10 min, washed with DPBS, and treated with $\mathrm{DT}^{\mathrm{pHAb}}(1 \mu \mathrm{M})$ for 30 min. The cells were washed with DMEM to remove $\mathrm{DT}^{\mathrm{pHAb}}$ and imaged. Panels from left to right represent the merge, $\mathrm{PS}^{\mathrm{TopFluor}}$ (green), and $\mathrm{DT}^{\mathrm{pHAb}}$ signals (red), respectively. Images of several adjacent endosomes are shown, with the one forming tubular structures indicated by a white arrow. Scale bars, $1 \mu \mathrm{m}$.

Movie S4. DT-induced vesicle budding and collapse from an enlarged endosome. HeLa cells were pretreated with YM201636 $(800 \mathrm{nM})$ for $2 \mathrm{~h}$ and DT ${ }^{\mathrm{TMR}}(5 \mu \mathrm{M})$ and Dextran ${ }^{\text {Alexa488 }}(50$ $\mu \mathrm{g} / \mathrm{mL}$ ) were added. After incubation for another $30 \mathrm{~min}$, the cells were washed and imaged by live-cell confocal microscopy every $4 \mathrm{~s}$. Panels from left to right represent the merge, Dextran $^{\text {Alexa488 }}$ (green), and DT ${ }^{\mathrm{TMR}}$ signals (red), respectively. The endosome undergoing vesicle budding and collapse is indicated by a white arrow. Scale bars, $1 \mu \mathrm{m}$.

Movie S5. NleC-induced vesicle budding and collapse in a HeLa cell. Cells were incubated with PS ${ }^{\text {TopFluor }}(1.5 \mu \mathrm{M})$ and $\mathrm{NleC}^{\mathrm{pHAb}}(5 \mu \mathrm{M})$ for 30 min, washed with DMEM, and imaged after $\sim 7 \mathrm{~min}$ at $\sim 8$-s intervals. The video shows the time-lapse images of a single endosome (boxed in Fig. 4A) during the period of 15-118 s after the initiation of imaging. Panels from left to right represent the merge, $\mathrm{PS}^{\mathrm{TopFluor}}$ (green), and $\mathrm{NleC}^{\mathrm{PHAb}}$ signals (red), respectively. A budded vesicle emerged from the endosome (indicated by an arrow) at $79 \mathrm{~s}$, separated from the endosome at 110 $\mathrm{s}$, and collapsed at $118 \mathrm{~s}$. Scale bars, $1 \mu \mathrm{m}$.

Movie S6. NleC-induced vesicle budding and collapse in a HeLa cell. Cells were incubated with PS ${ }^{\text {TopFluor }}(1.5 \mu \mathrm{M})$ and $\mathrm{NleC}^{\mathrm{pHAb}}(5 \mu \mathrm{M})$ for $30 \mathrm{~min}$, washed with DMEM, and imaged at 2-s intervals. The video shows the time-lapse images of a single endosome (shown in Figure S5 panel B) during the period of 25-42 s after the initiation of imaging. Panels from left to right represent the merge, $\mathrm{PS}^{\mathrm{TopFluor}}$ (green), and $\mathrm{NleC}^{\mathrm{pHAb}}$ signals (red), respectively. Scale bars, $1 \mu \mathrm{m}$. 